\title{
Tunable Narrow-Linewidth Ring Laser Based on the Polarization Conversion in a Tapered Fiber-Coupled Whispering Gallery Mode Resonator
}

\author{
Pengfa Chang ${ }^{1,2, *}$, Chen Wang ${ }^{1}$, Hong Han ${ }^{1}$, Feng Gao ${ }^{2, *} \mathbb{0}$, Ligang Huang ${ }^{3}$ and Anbang Wang ${ }^{1}$ \\ 1 Key Lab of Advanced Transducers \& Intelligent Control System, College of Physics \& Optoelectronic, \\ Ministry of Education and Shanxi Province, Taiyuan University of Technology, Taiyuan 030024, China; \\ wangchen1229@link.tyut.edu.cn (C.W.); hanhong@tyut.edu.cn (H.H.); wanganbang@tyut.edu.cn (A.W.) \\ 2 MOE Key Laboratory of Weak-Light Nonlinear Photonics, TEDA Applied Physics Institute and School of \\ Physics, Nankai University, Tianjin 300457, China \\ 3 Key Laboratory of Optoelectronic Technology \& Systems (Ministry of Education), Chongqing University, \\ Chongqing 400044, China; lghuang@cqu.edu.cn \\ * Correspondence: changpengfa@tyut.edu.cn (P.C.); fenggao@nankai.edu.cn (F.G.)
}

Citation: Chang, P.; Wang, C.; Han, H.; Gao, F.; Huang, L.; Wang, A. Tunable Narrow-Linewidth Ring Laser Based on the Polarization Conversion in a Tapered Fiber-Coupled Whispering Gallery Mode Resonator. Crystals 2021, 11, 1285. https://doi.org/10.3390/ cryst11111285

Academic Editors: Daquan Yang, Fei Xu, Jin-hui Chen and Sławomir Grabowski

Received: 10 September 2021

Accepted: 19 October 2021

Published: 23 October 2021

Publisher's Note: MDPI stays neutral with regard to jurisdictional claims in published maps and institutional affiliations.

Copyright: (c) 2021 by the authors. Licensee MDPI, Basel, Switzerland. This article is an open access article distributed under the terms and conditions of the Creative Commons Attribution (CC BY) license (https:// creativecommons.org/licenses/by/ $4.0 /)$.

\begin{abstract}
Based on the polarization conversion in a tapered fiber-coupled whispering gallery mode (WGM) system, a simple-structured narrow linewidth band-pass filter was fabricated and a narrow linewidth ring laser was demonstrated. With a fiber Bragg grating (FBG) to further select the resonant mode, the ring laser could be in the single-longitudinal-mode with the linewidth about $6 \mathrm{kHz}$ and its wavelength could be tuned from $1540.10 \mathrm{~nm}$ to $1570.01 \mathrm{~nm}$. Benefit from the dense modes in the WGM resonator, high conversion efficiency, and better stability of the structure, a tunable dual-wavelength laser could be achieved based on two FBGs with different resonant wavelengths. The configuration of the proposed laser is simple and stable, which will benefit its applications in the future.
\end{abstract}

Keywords: whispering gallery mode resonator; vector mode; add-drop filter; narrow-linewidth laser; all-fiber ring laser

\section{Introduction}

Single-longitudinal-mode (SLM) narrow-linewidth lasers have been widely used in areas such as coherent communication [1], metrology [2,3] and especially sensing applications [4-8]. Narrow linewidth indicates longer interference distance in various interferometers and SLM leads to better intensity stability. Besides these features, the mode-hop-free and large-tuning range are also important, which guarantee that the laser could be applied in the fine spectrum measurement. One of the most widely used configurations for such lasers is based on the Fabry-Perot (FP) interferometer, whose typical linewidth is about hundreds of kilohertz due to its low-quality factor (Q-factor). Considering the development of the whispering gallery mode (WGM) resonators in recent years [9-12], they have been widely used in the configuration of narrow-linewidth lasers due to their high Q-factor, which can be up to $10^{5}$ times larger than that of the FP cavity. The lasers developed include stimulated lasers [10,13], WGM resonator lasers [14,15], and various ring lasers [6,16]. Due to the advantages of relative lower cost and good tunability, ring lasers attract more attention among researchers. One of the key components in a ring laser is a band-pass filter. It is well known that a typical coupled WGM could provide a band-reject feature [17]. Although backscattering of the WGM could also provide a band-pass feature [14], the structures of the add-drop filter (ADF) based on a coupled WGM resonator system are more preferred for its high efficiency. The ADF filter based on the WGM includes at least two channels: one is through the channel, which usually provides the band-reject feature, and the other is the drop channel, which provides the band-pass feature. The channels could be either two 
individual waveguides $[15,16,18,19]$ or two modes in one waveguide [20-23]. Note that a waveguide has to be calibrated with the WGM within hundreds of nanometers to achieve high coupling efficiency. Therefore, the configuration with only one waveguide gains the advantages of simple structure, high efficiency, and high stability due to the quasi-critical coupling (QCC) effect of the WGM [24-26].

The first all-fiber ADF based on the linear polarization (LP) mode conversion of the WGM in a single tapered fiber (TF) was demonstrated in 2016, in which the $\mathrm{LP}_{11}$ modes in a multimode TF acted as a drop channel [20]. Although there was only one TF for the coupling of the WGM, an additional TF had to be introduced to couple the $\mathrm{LP}_{11}$ mode out to the realized band-pass feature, which made the structure not only vulnerable but also with large insertion loss $[20,27,28]$. With a tunable fiber Bragg grating (FBG) as a wide-band wavelength selector, an all-fiber SLM ring laser with a continuously tunable range had been demonstrated based on the structure [29]. The linewidth of the laser was as small as $3 \mathrm{kHz}$, which could fulfill most of its applications. The only problem was the complex structure of the ADF.

The latest works have shown that the polarization conversion in a TF could be realized by coupling it with a WGM, which could also be used to fabricate an ADF [21-23]. The configuration needed only one TF coupling with the WGM resonator and the insertion loss was negligible. The structure of the ADF was much simpler and the coupling state was more stable than the previous configurations [25]. It is a good candidate to realize the tunable ring lasers. Yet, due to the thinner TF applied, the coupling loss of the WGM to the TF increased compared to the multimode TF configuration, which increased the bandwidth of the filter. At the same time, in a ring laser, the polarization of the light will change its resonant wavelength; whether it can realize laser tuning still requires further demonstration.

In this article, we present an SLM narrow-linewidth laser with a widely tunable range based on the polarization conversion in a TF-coupled WGM system, which acted as a narrow band-pass filter in the laser. With a single FBG acting as the wavelength selector, the wavelength of the laser could be tuned continuously from $1540.10 \mathrm{~nm}$ to $1570.01 \mathrm{~nm}$ and a typical linewidth was $6 \mathrm{kHz}$ at the wavelength of $1550.17 \mathrm{~nm}$. With dual FBGs, the structure could also realize a configurable two-wavelength laser with more agility. The proposed laser gained advantages of narrow-linewidth, a widely tunable range, high efficiency, as well as robust and simple structure, which would be helpful for its future applications.

\section{Materials and Methods}

The fiber mode conversion may be realized by coupling two fiber modes to a WGM at the same time. These two fiber modes can be in different physics channels, which is the traditional ADF structure and has been studied universally, while they can also be in the same physics channel $[20,24]$. In previous work, it has been demonstrated that the cladding mode $\mathrm{LP}_{11}$ and the core mode $\mathrm{LP}_{01}$ could act as the drop mode and through mode of the $\mathrm{ADF}$, respectively. However, the $\mathrm{LP}_{11}$ mode had to be led out by an additional $\mathrm{TF}[27,28]$ with additional insertion loss to realize the band-pass feature of the ADF. The latest studies have shown that the orthogonal components of a vector mode in a single mode TF could serve the same function as well [21-23]. By a polarization coupler at the output port of the tapered fiber, the $\mathrm{HE}_{11} \mathrm{X}$ and $\mathrm{HE}_{11}{ }^{\mathrm{Y}}$ modes acting as the through mode and drop mode, respectively, could be separated simultaneously, and a convert efficiency at the drop mode, higher than $95 \%$, had been demonstrated with the QCC effect [25]. Although the configurations were totally different, all the structures could be explained by the same ADF theory $[18,20]$. For convenience, we explained the mechanism with the vector mode components, which act as the through mode and drop mode. The conversion efficiency of the drop mode is given as in [18,20,24]:

$$
D=\frac{4 \kappa_{X} \kappa_{Y}}{\left(\kappa_{0}+\kappa_{X}+\kappa_{Y}\right)^{2}}
$$


where $\kappa_{0}$ is the intrinsic loss of the WGM, $\kappa_{X}$ is the coupling loss to the through mode of the WGM, and $\kappa_{Y}$ is the coupling loss to the drop mode of the WGM. The conversion efficiency could be as high as approximately $100 \%$, i.e., $D=1$ when $\kappa_{X}=\kappa_{Y}>>\kappa_{0}[24,25]$.

Different from the mode conversion between the cladding modes and the core mode, the modes $\mathrm{HE}_{11} \mathrm{X}$ and $\mathrm{HE}_{11}{ }^{\mathrm{Y}}$ are degenerated and their mode fields are overlapped. Therefore, the decay rates of $\kappa_{X}$ and $\kappa_{Y}$ are nearly the same all the time [24,25]. According to previous research studies [30,31], the coupling losses between the two modes and a WGM obey the relationship of $\kappa_{X} / \kappa_{Y}=\cot ^{2} \theta$, where $\theta$ is the angle between the polarization directions of the WGM and the $\mathrm{HE}_{11}{ }^{\mathrm{X}}$ mode. It is obvious that when $\theta$ equals $45^{\circ}, \kappa_{X}$ will equal to $\kappa_{Y}$. With $\kappa_{X}>>\kappa_{0}$, the total transmission $D$ will be almost $100 \%$ according to Equation (1), which is called the ideal QCC state $[24,25]$. Note that the state will remain unchanged as the coupling gap varies when $\kappa_{X}$ is much larger than $\kappa_{0}$.

The configuration to realize the polarization conversion is shown in Figure 1a. A polarizer P1 is placed before the input port of the TF. When the pump light propagates through $P 1$, it will represent linear polarization and be defined as $\mathrm{HE}_{11} \mathrm{X}$. After the WGM resonator, the output light will convert to the orthogonal vector mode $\mathrm{HE}_{11}{ }^{\mathrm{Y}}$ with suitable configuration. The light that does not couple with the cavity modes will remain in mode $\mathrm{HE}_{11} \mathrm{X}$. With a second polarizer $\mathrm{P} 2$ at the output port, the device could be regarded as a narrow linewidth polarization filter (PF). Note that the feature of the output spectra is determined by the polarization direction of P2 [25]. Either the band-pass or band-reject feature is available. Since we expect a band-pass filter for a ring laser, the polarization direction of P2 should be perpendicular to that of the P1. As an example, a band-pass spectrum of the PF is shown in Figure 1b. To further select the drop channels, an FBG with a circulator is introduced, identical to that in the previous work [29], and the transmission spectrum at the end of the band-pass filter will be the combination spectrum of the FBG and the drop mode, as shown in Figure $1 \mathrm{~b}$. The bandwidth of a typical FBG is about $0.2 \mathrm{~nm}$, which could include several WGMs at the same time. When there is competition among the modes, only one mode could actually work in the ring laser, which is usually the mode with highest conversion efficiency. As an example, a working spectrum of the FBG is presented arbitrarily as a blue inverse U-shape line in Figure 1b, and the mode with the highest conversion efficiency within the spectrum of the FBG will be selected, which is marked as mode 1 . When the FBG is stretched by external force, the center wavelength of its spectrum will red-shift and the activated mode will change accordingly, which is shown as a dotted blue line and labelled as mode 2 in Figure $1 \mathrm{~b}$ as an example. The situation will be the same when another FBG is introduced, which is shown as the second blue inverse U-shape line and labelled as mode 3.

(a)

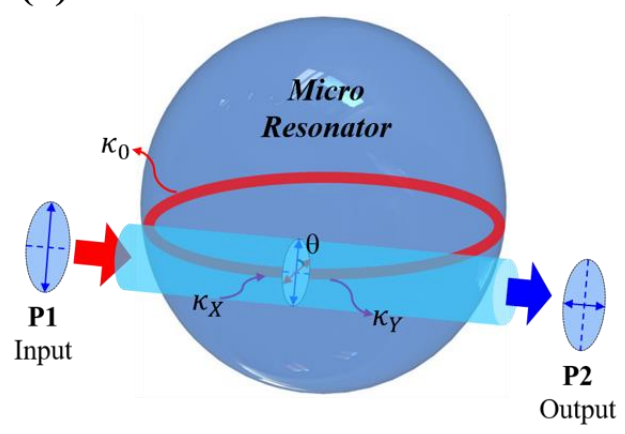

(b)

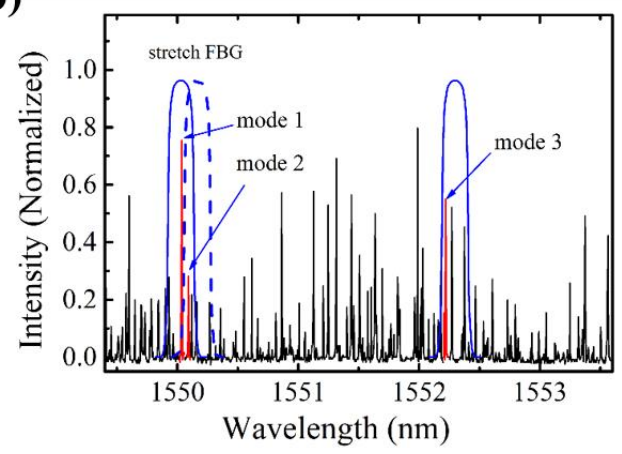

Figure 1. (a) Schematic diagram of the polarization conversion based on orthogonal modes coupled a WGM resonator, including P1 and P2, which are the polarizers. (b) The principle of the mode selection brought by the FBG. The black line is the spectrum of the drop mode of the polarization conversion device, the blue inverse U-shape lines indicate the reflection spectrum of FBG, and the red modes 1, 2, and 3 are the WGMs selected by the FBG. 


\section{Experiment and Results}

In the experiment, a microsphere was fabricated from a fiber tip with a fiber fusion splicer to act as a WGM resonator whose diameter was about $215 \mu \mathrm{m}$. The typical $Q-$ value of the WGM applied was about $10^{7}$, corresponding to a bandwidth of $20 \mathrm{MHz}$. The waist of the TF was about $1.6 \mu \mathrm{m}$. The coupling gap between the microsphere and the $\mathrm{TF}$ could be precisely modified by a 3D nano-positioning system. The polarizer before the TF was placed inside a fiber bench (FB) whose extinction ratio was better than $30 \mathrm{~dB}$. The polarizer after the TF was replaced with a polarization beam splitter (PBS) whose polarization directions at its outputs were orthogonal. To achieve a high efficiency of the band-pass filter, the polarization direction of the WGM needed to be calibrated. With the microsphere tuned in the under-coupled regime, we tuned the polarizer in the fiber bench to change the polarization of the input light until the coupling efficiency of the WGM reached its maximum. After the calibration, the polarizations of the input light and the WGM were taken as parallel and such a state of the input light was defined as $\theta=0^{\circ}$. Then, the microsphere was shifted away from the TF and the polarizer was rotated about $45^{\circ}$ from the position of $\theta=0^{\circ}$. After that, a PBS with a polarization controller was connected to the output port of the TF. By tuning a polarization controller between the PBS and TF, the light in one of its ports was minimized, defined as the drop port, and the other one was defined as the through port. Then, the microsphere was shifted back. With a tunable laser sweeping its wavelength at the input and with a pair of photodetectors (PDs) at the outputs to monitor the transmissions, both spectra at the through port and drop port could be measured by an oscilloscope (OSC), which are shown in Figure 2a,b, respectively. The transmission spectrum of the through mode included a serial of notches, while the transmission spectrum of the drop modes included a serial of peaks. Within 1550-1551 $\mathrm{nm}$, there were more than 20 modes whose conversion efficiency was larger than $10 \%$, as depicted in Figure 2b, and the average spectra adjacent to them was about $0.05 \mathrm{~nm}$. The conversion efficiency could be very high under suitable conditions. As an example, one peak with a conversion efficiency above $99 \%$ is marked in Figure $2 \mathrm{~b}$. In allowing for the insertion loss to extract the drop mode, the configuration gained much better conversion efficiency than in previous works [20,24].
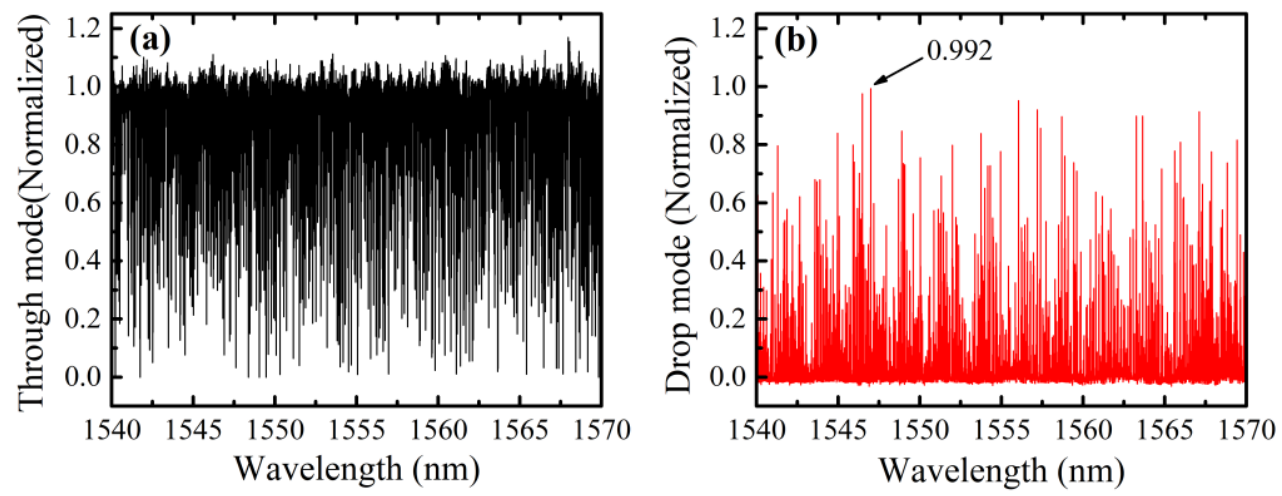

Figure 2. $(\mathbf{a}, \mathbf{b})$ are the transmission spectra of the through mode and drop mode in the wavelength range of $1540 \mathrm{~nm}$ to $1570 \mathrm{~nm}$.

With the filter, a typical ring laser setup was applied in the experiment. The bandpass filter could provide a series of band-pass peaks with a bandwidth of about tens of MHz. As shown in Figure 3, an FBG was used as the additional wavelength selector and could provide an extended tuning range. An erbium-doped fiber amplifier (EDFA) was used as the gain medium. An isolator was used to ensure unidirectional emission, which could avoid both the spatial hole-burning and non-linear back-scattering. A polarization controller was used to optimize the output laser. With a 99/1 optical coupler, $99 \%$ of the power would circulate within the ring cavity and $1 \%$ would be led out. The output power was divided by a 10/90 coupler, in which the smaller part was led to an optical 
spectrum analyzer (OSA) to monitor both wavelength and power level and the rest was led into the delayed self-heterodyne interferometer (DSHI) device, which was usually used to characterize the linewidth of the laser [32,33]. In the DSHI device, an acousto-optic tunable add/drop coupler (AOTC) was used as the frequency shift generator, with a frequency shift of about $0.9 \mathrm{MHz}$ [29]. The delay line was about $40 \mathrm{~km}$ with a delay time of about $200 \mu \mathrm{s}$, which could provide a resolution of around $5 \mathrm{kHz}$ in the linewidth measurement. The beat generated by the interferometer was recorded by an OSC via a PD.

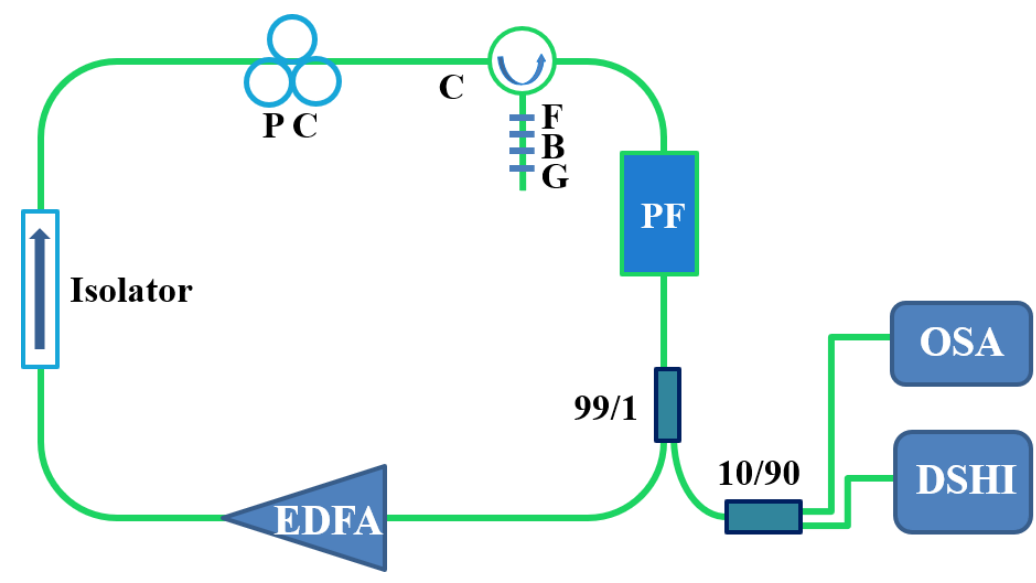

Figure 3. The diagram of the ring laser. Abbreviations: PC, polarization controller; $\mathrm{C}$, optical circulator; FBG, fiber Bragg grating; PF, polarization filter shown as the inset, which has been introduced above; FB, fiber bench; PBS, polarization beam splitter; EDFA, erbium-doped fiber amplifier; isolator, optical isolator; $99 / 1$ and 10/90, optical couplers and their ratios; OSA, optical spectrum analyzer; and DSHI, delayed self-heterodyne interferometer device.

A typical result with an arbitrary wavelength of $1550.17 \mathrm{~nm}$ is shown in Figure 4, with comparable SNR to previous work [29]. The spectrum is shown in Figure 4a and the frequency spectrum of the optical beat from the DSHI is shown in Figure $4 \mathrm{~b}$. In the figure, the main peak at $0.9 \mathrm{MHz}$ was from the optical beat. There were also peaks brought by the frequency multiplication, such as the peaks at $1.8 \mathrm{MHz}, 2.7 \mathrm{MHz}, 3.6 \mathrm{MHz}$, and 4.5 MHz. The 3-dB bandwidth of the peak at $0.9 \mathrm{MHz}$ was about $12 \mathrm{kHz}$ with Lorentz fitting, as shown in the inset of Figure $4 \mathrm{~b}$. According to the theory, the linewidth of the laser should be about $6 \mathrm{kHz}[32,33]$. The length of the ring cavity was about $40 \mathrm{~m}$ and the frequency beat from the side longitudinal mode should be located at $5 \mathrm{MHz}$ accordingly, which could hardly be observed in the measurement, as shown in the position of the blue circle in Figure $4 \mathrm{~b}$. Therefore, the laser in the experiment worked in the single-longitudinalmode state.
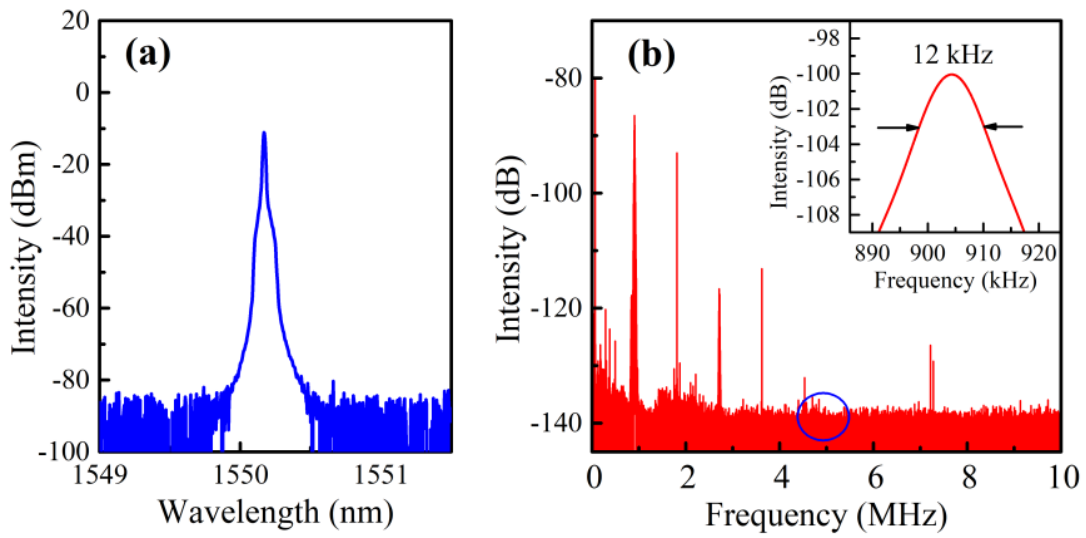

Figure 4. (a) The spectrum of the output laser. (b) The measurement result of the linewidth by the DSHI device. 
As in previous work, the wavelength of the laser could be tuned [29]. By stretching the FBGs with different central wavelengths, the wavelength of the laser could be tuned coarsely and it covered the range of $1540.10 \mathrm{~nm}$ to $1570.01 \mathrm{~nm}$ with the step of $0.12 \mathrm{~nm}$, which is shown in Figure 5a,b. During the coarse tuning, the output power would fluctuate within $5 \mathrm{~dB}$, which originated from the difference in the polarization mode conversion efficiency in the WGM-based filter, as shown in Figure $2 b$. With specially designed FBG, the tuning range of a single FBG could be expanded beyond $40 \mathrm{~nm}$ [34], which could simplify the structure further.
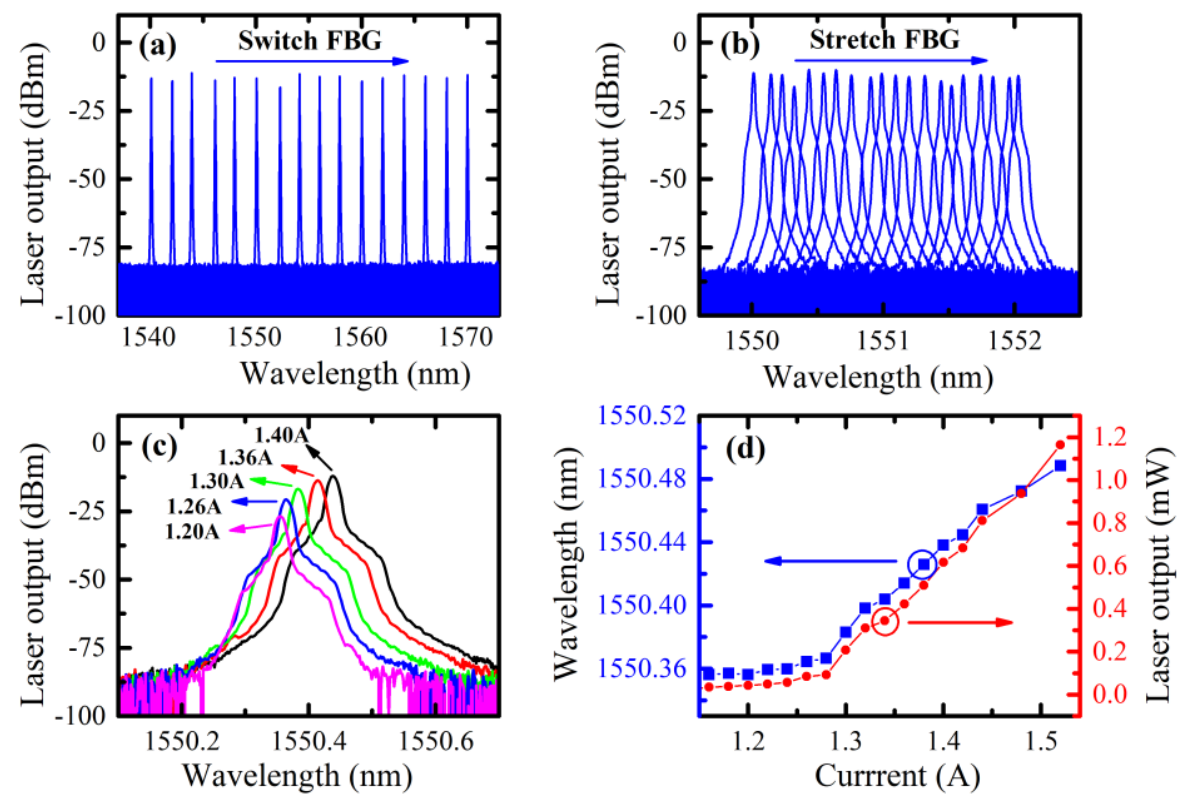

Figure 5. The tunability of the laser. (a) The output lasers based on the FBGs with different resonance wavelengths. (b) The output lasers based on different WGMs when stretching the FBG. (c) The output spectrum of the laser when changing the pump-current in the EDFA. (d) The wavelengths and output intensities at different pump-currents.

The fine continuous tuning was based on the optical-thermo effect of the WGM. In a high-Q WGM, the refractive index and volume of the WGM would change at different pump-powers due to the changing of the temperature, which would lead to the changing of the resonance wavelength [16,35]. Therefore, the wavelength of the laser could be tuned continuously by adjusting the pump power of the ring cavity [29]. One of the examples is shown in Figure 5c. By changing the pump current from $1.20 \mathrm{~A}$ to $1.50 \mathrm{~A}$ in the EDFA, the wavelength of the output laser could be fine-tuned from $1550.36 \mathrm{~nm}$ to $1550.49 \mathrm{~nm}$ without mode hop. Note that with a suitable pump-current of the EDFA, the tuning was approximately linear, for example, from $1.3 \mathrm{~A}$ to $1.5 \mathrm{~A}$, as shown in Figure $5 \mathrm{~d}$.

Since the longitudinal mode of the ring laser could be selected by an FBG, it is simple to realize a multi-wavelength laser with additional FBGs introduced. As an example, we demonstrated a dual-wavelength laser. In the configuration, two FBGs connected with a 50:50 coupler had been inserted in the optical ring laser via a circulator and each one was connected with a polarization controller to adjust the polarization of the reflected light from the FBG, as shown in Figure 6a. When the resonant wavelength of the FBG was changed by different stress, the wavelengths of the laser could be modified accordingly, as shown in Figure 6b. In the figure, one of the wavelengths was fixed at $1550.07 \mathrm{~nm}$, while the other was tuned from $1550.61 \mathrm{~nm}$ to $1552.21 \mathrm{~nm}$. The wavelength spacing was changed from $0.54 \mathrm{~nm}$ to $2.14 \mathrm{~nm}$. 

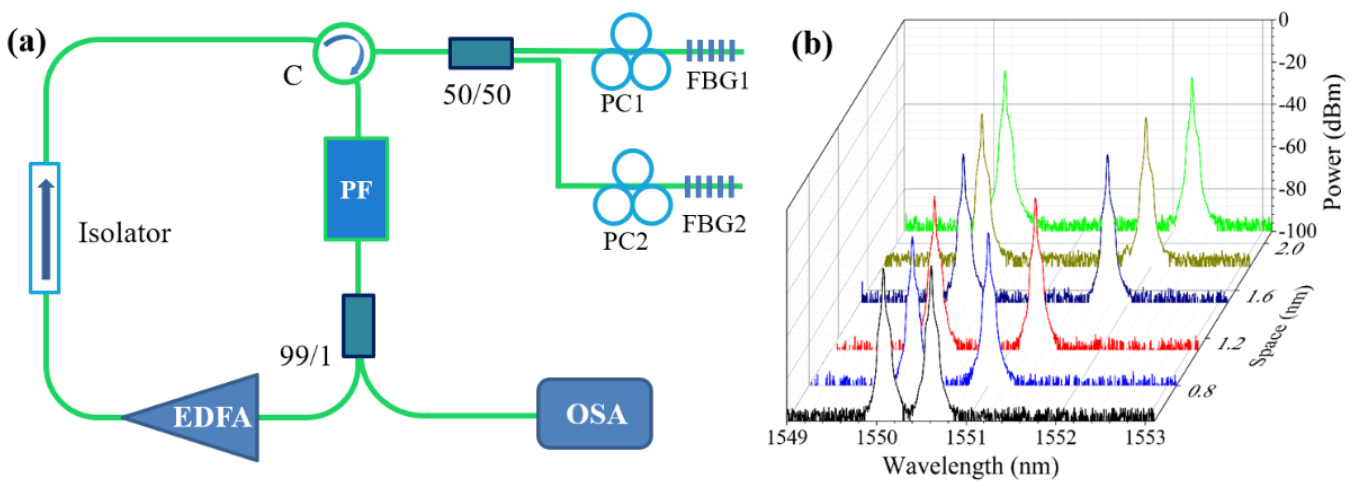

Figure 6. (a) Diagram of the dual-wavelength laser. Abbreviations: PC1 and PC2, polarization controllers; C, optical circulator; FBG1 and FBG2: fiber Bragg gratings; PF, polarization filter shown as the inset, which has been introduced in Figure 3; EDFA, erbium-doped fiber amplifier; isolator, optical isolator; 50/50 and 99/1, optical couplers and their ratios; and OSA, optical spectrum analyzer. (b) The tunability of the dual-wavelength laser.

\section{Conclusions}

In summary, we demonstrated an all-fiber SLM narrow-linewidth ring laser based on a WGM resonator and FBGs whose linewidth was about $6 \mathrm{kHz}$. Only one TF was needed in the band-pass filter with a QCC-state, which made the structure simple, stable, and effective. By inserting an FBG as an additional wavelength selector, the wavelength of the laser could be stabilized and selected simultaneously. The output wavelength of the laser could be continuously tuned, covering the whole range from $1540.10 \mathrm{~nm}$ to $1570.01 \mathrm{~nm}$. Through the benefit of the dense modes and high conversion efficiency of the device, a dual-wavelength laser could also be achieved and the wavelength spacing was changed form $0.54 \mathrm{~nm}$ to $2.14 \mathrm{~nm}$ by adjusting the stress on the FBGs and the polarization of the PCs. Compared with the previous work [29], this work sustained the advantages of all-fiber structures as well as the SLM and widely tunable range, with a comparable linewidth and SNR. With a simpler structure, alongside better efficiency and stability, the laser is more practical and has significant potential applications in the future.

Author Contributions: Conceptualization, P.C.; data curation, P.C. and C.W.; funding acquisition, P.C. and A.W.; investigation, P.C., C.W., and L.H.; methodology, P.C.; project administration, F.G. and A.W.; writing—original draft preparation, P.C.; writing—review and editing, P.C., C.W., H.H., F.G., and L.H. All authors have read and agreed to the published version of the manuscript.

Funding: This study received funding from the National Science Foundation of China (NSFC; 12074199, 62105233, 61805170, 61822509, 61731014, 61671316, 61805171, and 61705160); National Cryptography Foundation (MMJJ20170207); International Cooperation Program and Natural Science Foundation of Shanxi (201603D421008, 201801D221189, 201701D1211362, and 201802044); and Open fund of MOE Key Laboratory of Weak-Light Nonlinear Photonics, (Nankai University), grant number OS 21-2.

Data Availability Statement: The data presented in this study are available upon request from the corresponding author.

Conflicts of Interest: The authors declare no conflict of interest.

\section{References}

1. Han, Y.; Li, G. Coherent optical communication using polarization multiple-input-multiple-output. Opt. Express 2005, 13, 7527-7534. [CrossRef]

2. Geng, J.; Spiegelberg, C.; Jiang, S. Narrow linewidth fiber laser for 100-km optical frequency domain reflectometry. IEEE Photonics Technol. Lett. 2005, 17, 1827-1829. [CrossRef]

3. Pan, S.; Yao, J. A wavelength-switchable single-longitudinal-mode dual-wavelength erbium-doped fiber laser for switchable microwave generation. Opt. Express 2009, 17, 5414-5419. [CrossRef] 
4. Juarez, J.C.; Maier, E.W.; Choi, K.N.; Taylor, H.F. Distributed fiber-optic intrusion sensor system. J. Lightwave Technol. 2005, 23, 2081-2087. [CrossRef]

5. Francois, A.; Himmelhaus, M. Optical biosensor based on whispering gallery mode excitations in clusters of microparticles. Appl. Phys. Lett. 2008, 92, 141107. [CrossRef]

6. Conti, G.N.; Berneschi, S.; Barucci, A.; Cosi, F.; Soria, S.; Trono, C. Fiber ring laser for intracavity sensing using a whisperinggallery-mode resonator. Opt. Lett. 2012, 37, 2697-2699. [CrossRef] [PubMed]

7. Wang, L.; Chandrahalim, H.; Zhou, J.; Li, Z.; Chen, C.; Cho, S.; Zhang, H.; Mei, T.; Tian, H.; Oki, Y.; et al. Demonstration of versatile whispering-gallery micro-lasers for remote refractive index sensing. Opt. Express 2018, 26, 5800-5809.

8. Lai, Y.H.; Suh, M.G.; Lu, Y.K.; Shen, B.; Yang, Q.F.; Wang, H.; Li, J.; Lee, S.H.; Yang, K.Y.; Vahala, K. Earth rotation measured by a chip-scale ring laser gyroscope. Nat. Photonics 2020, 14, 345-350. [CrossRef]

9. Gorodetsky, M.L.; Savchenkov, A.A.; Ilchenko, V.S. Ultimate Q of optical microsphere resonators. Opt. Lett. 1996, $21,453-455$. [CrossRef]

10. Spillane, S.M.; Kippenberg, T.J.; Vahala, K.J. Ultralow-threshold Raman laser using a spherical dielectric microcavity. Nature 2002, 415, 621-623. [CrossRef] [PubMed]

11. Vahala, K.-J. Optical microcavities. Nature 2003, 424, 839-846. [CrossRef] [PubMed]

12. Lee, H.; Chen, T.; Li, J.; Yang, K.-Y.; Jeon, S.; Painter, O.; Vahala, K.-J. Chemically etched ultrahigh-Q wedge-resonator on a silicon chip. Nat. Photonics 2012, 6, 369-373. [CrossRef]

13. Grudinin, I.S.; Matsko, A.B.; Maleki, L. Brillouin lasing with a CaF2 Whispering Gallery Mode Resonator. Phys. Rev. Lett. 2009, 102, 043902. [CrossRef]

14. Kieu, K.; Mansuripur, M. Fiber laser using a microsphere resonator as a feedback element. Opt. Lett. 2007, 32, 244-246. [CrossRef]

15. Rivera-Pérez, E.; Díez, A.; Andrés, M.V.; Cruz, J.L.; Rodríguez-Cobos, A. Tunable narrowband fiber laser with feedback based on whispering gallery mode resonances of a cylindrical microresonator. Opt. Lett. 2013, 38, 1636-1638. [CrossRef] [PubMed]

16. Sprenger, B.; Schwefel, H.G.L.; Wang, L.J. Whispering-gallery-mode-resonator-stabilized narrow-linewidth fiber loop laser. Opt. Lett. 2009, 34, 3370-3372. [CrossRef] [PubMed]

17. Knight, J.C.; Cheung, G.; Jacques, F.; Birks, T.A. Phase-matched excitation of whispering-gallery-mode resonances by a fiber taper. Opt. Lett. 1997, 22, 1129-1131. [CrossRef]

18. Monifi, F.; Friedlein, J.; Ozdemir, S.K.; Lan, Y. A robust and tunable add-drop filter using whispering gallery mode microtoroid resonator. J. Lightwave Technol. 2012, 30, 3306-3315. [CrossRef]

19. Collodo, M.C.; Sedlmeir, F.; Sprenger, B.; Svitlov, S.; Wang, L.J.; Schwefel, H.G.L. Sub-kHz lasing of a CaF 2 whispering gallery mode resonator stabilized fiber ring laser. Opt. Express 2014, 22, 19277-19283. [CrossRef]

20. Huang, L.G.; Wang, J.; Peng, W.H.; Zhang, W.D.; Bo, F.; Yu, X.Y.; Gao, F.; Chang, P.F.; Song, X.B.; Zhang, G.Q.; et al. Mode conversion in a tapered fiber via a whispering gallery mode resonator and its application as add/drop filter. Opt. Lett. 2016, 41, 638-641. [CrossRef] [PubMed]

21. Melloni, A.; Morichetti, F.; Martinelli, M. Polarization conversion in ring resonator phase shifters. Opt. Lett. 2004, 29 , 2785-2787. [CrossRef]

22. Bianucci, P.; Fietz, C.R.; Robertson, J.W.; Shvets, G.; Shih, C.-K. Polarization conversion in a silica microsphere. Opt. Express 2007, 15, 7000-7005. [CrossRef]

23. Bianucci, P.; Fietz, C.R.; Robertson, J.W.; Shvets, G.; Shih, C.-K. Whispering gallery mode microresonators as polarization converters. Opt. Lett. 2007, 32, 2224-2226. [CrossRef]

24. Li, X.T.; Chang, P.F.; Huang, L.G.; Gao, F.; Zhang, W.D.; Bo, F.; Zhang, G.Q.; Xu, J.J. Quasicritical coupling in a few-mode tapered-fiber coupled whispering-gallery-mode system. Phys. Rev. A 2018, 98, 053814. [CrossRef]

25. Chang, P.F.; Cao, B.T.; Gao, F.; Huang, L.G.; Zhang, W.D.; Bo, F.; Yu, X.Y.; Zhang, G.Q.; Xu, J.J. Enhance stable coupling region of a high-Q WGM up to micrometer. Appl. Phys. Lett. 2019, 115, 211104. [CrossRef]

26. Li, J.; Hu, Y.; Gan, X.; Gao, F.; Zhang, W.; Huang, L.; Bo, F.; Zhang, G.; Xu, J. Bandwidth tunable filter based on ideal quasi-critical coupling state in WGM cavity. J. Lightwave Technol. 2021, 39, 6547-6552. [CrossRef]

27. Zhang, W.D.; Huang, L.G.; Gao, F.; Bo, F.; Xuan, L.; Zhang, G.Q.; Xu, J.J. Tunable add/drop channel coupler based on an acousto-optic tunable filter and a tapered fiber. Opt. Lett. 2012, 37, 1241-1243. [CrossRef]

28. Zhang, W.D.; Huang, L.G.; Gao, F.; Bo, F.; Zhang, G.Q.; Xu, J.J. Tunable broadband light coupler based on two parallel all-fiber acousto-optic tunable filters. Opt. Express 2013, 21, 16621-16628. [CrossRef] [PubMed]

29. Huang, L.G.; Chang, P.F.; Li, X.T.; Zhang, W.D.; Li, Y.J.; Gao, F.; Bo, F.; Gao, L.; Huang, W.; Liu, M.; et al. All-fiber narrow-linewidth ring laser with continuous and large tuning range based on microsphere resonator and fiber Bragg grating. Opt. Express 2018, 26, 32652-32661. [CrossRef] [PubMed]

30. Chang, P.F.; Cao, B.T.; Huang, L.G.; Li, J.W.; Hu, Y.; Gao, F.; Zhang, W.D.; Bo, F.; Yu, X.Y.; Zhang, G.Q.; et al. Polarization-modified Fano line shape spectrum with a single whispering gallery mode. Sci. China Phys. Mech. Astron. 2020, 63, 214211. [CrossRef]

31. Li, B.B.; Xiao, Y.F.; Zou, C.-L.; Liu, Y.-C.; Jiang, X.-F.; Chen, Y.-L.; Li, Y.; Gong, Q.H. Experimental observation of Fano resonance in a single whispering-gallery microresonator. Appl. Phys. Lett. 2011, 98, 021116. [CrossRef]

32. Okoshi, T.; Kikuchi, K.; Nakayama, A. Novel method for high resolution measurement of laser output spectrum. Electron. Lett. 1980, 16, 630-631. [CrossRef] 
33. Iwatsuki, K.; Okamura, H.; Saruwatari, M. Wavelength-tunable single-frequency and single-polarisation Er-doped fibre ring-laser with $1.4 \mathrm{kHz}$ linewidth. Electron. Lett. 1990, 26, 2033-2035. [CrossRef]

34. Song, Y.W.; Havstad, S.A.; Starodubov, D.; Xie, Y.; Willner, A.E.; Feinberg, J. 40-nm-wide tunable fiber ring laser with single-mode operation using a highly stretchable FBG. IEEE Photonics Technol. Lett. 2001, 13, 1167-1169. [CrossRef]

35. Carmon, T.; Yang, L.; Vahala, K.J. Dynamical thermal behavior and thermal self-stability of microcavities. Opt. Express 2004, 12, 4742-4750. [CrossRef] 